\title{
Structure of graphene and a surface carbide grown on the (0001) surface of rhenium
}

\author{
Estelle Mazaleyrat, ${ }^{1,2}$ Monica Pozzo $\odot,{ }^{3}$ Dario Alfè, ${ }^{3,4}$ Alexandre Artaud, ${ }^{1,2}$ Ana Cristina Gómez Herrero, ${ }^{1}$ Simone Lisi, \\ Valérie Guisset, ${ }^{1}$ Philippe David, ${ }^{1}$ Claude Chapelier, ${ }^{2}$ and Johann Coraux (1) ${ }^{1, *}$ \\ ${ }^{1}$ Univ. Grenoble Alpes, CEA, IRIG/DEPHY/PHELIQS, 38000 Grenoble, France \\ ${ }^{2}$ Univ. Grenoble Alpes, CNRS, Grenoble INP, Institut Néel, 38000 Grenoble, France \\ ${ }^{3}$ Department of Earth Sciences, London Centre for Nanotechnology and Thomas Young Centre at University College London, \\ University College London, Gower Street, London WC1E 6BT, United Kingdom \\ ${ }^{4}$ Dipartimento di Fisica Ettore Pancini, Università di Napoli Federico II, Monte Sant'Angelo, I-80126 Napoli, Italy
}

(Received 6 August 2020; accepted 17 November 2020; published 8 December 2020)

\begin{abstract}
Transition metal surfaces catalyze a broad range of thermally activated reactions involving carbon-containing species, from atomic carbon to small hydrocarbons or organic molecules, and polymers. These reactions yield well-separated phases, for instance graphene and the metal surface, or, on the contrary, alloyed phases, such as metal carbides. Here, we investigate carbon phases on a rhenium (0001) surface, where the former kind of phase can transform into the latter. We find that this transformation occurs with increasing annealing time, which is hence not suitable to increase the quality of graphene. Our scanning tunneling spectroscopy and reflection high-energy electron diffraction analysis reveal that repeated short annealing cycles are best suited to increase the lateral extension of the structurally coherent graphene domains. Using the same techniques and with the support of density functional theory calculations, we next unveil, in real space, the symmetry of the many variants (two sixfold families) of a rhenium surface carbide observed with diffraction since the 1970s, and finally propose models of the atomic details. One of these models, which nicely matches the microscopy observations, consists of parallel rows of eight aligned carbon trimers with a so-called $(7 \times \sqrt{19})$ unit cell with respect to $\operatorname{Re}(0001)$.
\end{abstract}

DOI: 10.1103/PhysRevMaterials.4.124002

\section{INTRODUCTION}

Graphene growth on strongly interacting metallic substrates such as $\operatorname{Re}(0001)$ [1-5], $\operatorname{Ru}(0001)$ [6-9], $\operatorname{Rh}(111)$ [10], and $\mathrm{Ni}(111)$ [11] has the advantage of selecting one crystallographic orientation of graphene with respect to its substrate. This is related with a tendency to form covalent bonds between graphene and the substrate $[5,10]$, as opposed to graphene grown on weakly interacting metallic substrates such as $\operatorname{Ir}(111)$ [12,13], $\operatorname{Pt}(111)$ [14-16], Cu(111) [17], $\mathrm{Ag}(111)$ [18], and $\mathrm{Au}(111)$ [19], where van der Waals bonding is dominant $[5,10,13]$. Graphene growth on the latter kind of substrates results in domains with a large number of possible crystallographic orientations with respect to the substrate [14-17,20-23], which is problematic in the prospect of the production of highly ordered graphene.

After its growth with a well-defined crystallographic orientation on strongly interacting metallic substrates, singlelayer graphene has been successfully transferred to other, application-ready substrates [24-27]. Despite these successes, a number of difficulties associated with the use of these substrates must be borne in mind, since they are not easily overcome. In particular, on $\operatorname{Rh}(111), \mathrm{Ni}(111)$, and $\operatorname{Re}(0001)$ surfaces, there is a subtle competition between the formation of different carbon phases, graphene, and metal carbides [2,28-31]. The relative stability of these phases depends on

\footnotetext{
*johann.coraux@neel.cnrs.fr
}

the temperature. In addition, even when in some temperature range graphene is the most stable species on the surface, its nucleation may require a high carbon chemical potential (a high carbon adatom concentration) [29], without which the other carbon phases exclusively form on the surface. In the case of $\operatorname{Re}(0001)$, which is at the same time a growth substrate and a platform to induce superconductivity up to $2.1 \mathrm{~K}$ in graphene by a proximity effect [3] (the strong graphene$\mathrm{Re}$ interaction destroying the unique electronic spectrum of graphene can be suppressed while maintaining the proximity effect using Au intercalation [32]), selective growth of graphene is limited to a narrow window of growth parameters (substrate temperature, hydrocarbon gas pressure, and exposure time) [2].

Although obviously considered as detrimental in the context of graphene production, the surface metal carbides that sometimes grow at the expense of graphene provide wellordered surface structures with crystallographic unit cells at the scale of nanometers. This makes them efficient nanopatterns driving the self-organization of functional clusters, especially metallic clusters of interest for magnetic data storage or heterogeneous catalysis applications [33-35].

Only a few works devoted to surface carbides formed on the (0001) surface of rhenium have been reported so far $[2,30,36]$. An ordered carbide overlayer was previously identified with low-energy electron diffraction (LEED) [2,36], from which unit cell vectors of $7 \vec{a}_{\mathrm{Re}, 1}$ and $2 \vec{a}_{\mathrm{Re}, 1}+5 \vec{a}_{\mathrm{Re}, 2}$ [with $\vec{a}_{\mathrm{Re}, 1}, \vec{a}_{\mathrm{Re}, 2}$ the $\operatorname{Re}(0001)$ unit vectors] were inferred. In the extended Wood's notation [23], this superstructure corresponds 
to $(7 R 0 \times \sqrt{19} R(-23.4))$, and is referred to as $(7 \times \sqrt{19})$ $[2,36]$. It was never observed directly with a local probe method.

Here, we investigate the structure of graphene and the $(7 \times \sqrt{19})$ surface carbide grown on $\operatorname{Re}(0001)$. To characterize lattice parameters and learn about the symmetry of these carbon phases, we used an ensemble-averaging in operando probe, reflection high-energy electron diffraction (RHEED). A local probe analysis using scanning tunneling microscopy (STM) is less suited for this purpose, as it reveals, for instance, slight spatial structural variations about the average structure of graphene/Re(0001) [3,23]. However, STM was used to get insights into the graphene and carbide morphologies and to resolve the atomic details of the carbide with the help of density functional theory (DFT) calculations. We find that increasing the number of annealing cycles during graphene growth increases the quality and extension of graphene domains. As is known, excessive annealing temperatures promote the formation of the $(7 \times \sqrt{19})$ surface carbide, which we observed for the first time by STM. The carbide has various crystallographic orientations on the surface and exists in the form of six kinds of domains, each with a specific atomic arrangement. Four atomic models, complying with former $\mathrm{x}$-ray photoelectron spectroscopy (XPS) characterization, are proposed for the carbide structure of one of these domains, whose relative stability is probed with DFT calculations. The models are low-density $\mathrm{C}$ phases (considering the topmost $\mathrm{Re}$ layer, a $\operatorname{Re}_{11} \mathrm{C}_{8}$ compound) compared to graphene/Re(0001); they consist of a layer of parallel rows of $\mathrm{C}$ trimers attached to the hollow binding sites of the substrate, which experiences significant lattice distortions in its topmost layers.

\section{MATERIALS AND METHODS}

The surface preparation of the $\operatorname{Re}(0001)$ single crystal substrate (see details in the Supplemental Material (SM) [37]) and the subsequent growth of graphene and of the carbide were performed under ultrahigh vacuum (UHV), in a chamber having a base pressure of $10^{-10}$ mbar.

Graphene was grown using the method described in Ref. [2], which consists of a succession of rapid annealing cycles under $\mathrm{C}_{2} \mathrm{H}_{4}$ atmosphere $\left(\mathrm{C}_{2} \mathrm{H}_{4}\right.$ introduced in the UHV chamber via a leak valve). The $\operatorname{Re}(0001)$ surface was first exposed to an ethylene pressure $P_{\mathrm{C}_{2} \mathrm{H}_{4}}$ of 1 to $5 \times 10^{-7} \mathrm{mbar}$, at room temperature. This is expectedly a way to reach a highenough carbon surface concentration for graphene nucleation after the temperature is increased in the next step (exposing the surface to $\mathrm{C}_{2} \mathrm{H}_{4}$ only after the temperature has been increased does not allow the formation of graphene), similarly to the case of graphene $/ \mathrm{Rh}(111)$ [29]. The temperature was next rapidly increased to 870-970 K (measured with a Keller infrared pyrometer assuming an emissivity of 0.3 ), and then slowly decreased to $520 \mathrm{~K}$. This cycle was repeated several times. During this process, it is expected that the graphene coverage approaches $100 \%$ already after the few first cycles, since the ethylene precursor is provided at the metal surface held at elevated temperatures. The cycling process is thought to increase the structural quality of graphene thanks to the high-temperature (870-970 K) treatment, while limiting the decomposition of graphene via C-C bond breaking that would be promoted by increasing time spent at elevated temperature [2]. After the temperature cycles under ethylene partial pressure, a final annealing cycle was performed in absence of $\mathrm{C}_{2} \mathrm{H}_{4}$. Depending on the duration of this last step, part of the graphene was transformed into rhenium carbide.

During the exposure to $\mathrm{C}_{2} \mathrm{H}_{4}$ and thermal treatment, snapshots of the RHEED patterns (10 keV electron source) produced by the surface were acquired at a rate of typically $1 \mathrm{~Hz}$ using a CCD camera $(1280 \times 960$ pixels $)$. The structural analysis consisted of extracting the position and full width at half maximum (FWHM) of the diffraction streaks in the RHEED patterns, giving information on the average lattice parameter and sizes of the corresponding structurally coherent domains, respectively. The extraction relied on fits of the experimental streak profiles extracted within the rectangular regions of interest displayed in Fig. 1(a), using a set of Lorentzians. More details are given in the SM, Fig. S2 in particular [37].

STM measurements were performed at room temperature before and after one or several temperature cycles with the samples transferred under UHV to a commercial Omicron UHV-STM 1, with W chemically etched tips.

Calculations were performed using DFT $[38,39]$, with exchange-correlation effects included at the level of the PBEGGA [40] functional. We used the projector augmented wave [41] (PAW) method as implemented in VASP $[42,43]$ to account for the core electrons of both $\mathrm{Re}$ and $\mathrm{C}$ atoms, with the $6 s$ and $5 d$ electrons of Re and the $2 s$ and $2 p$ electrons of $\mathrm{C}$ explicitly included in the valence band. To represent the KohnSham orbitals we used a plane-wave expansion with a kinetic energy cutoff of $400 \mathrm{eV}$. As the calculations are fully periodic in the three spatial directions, surfaces have been represented in the usual slab geometry, with 4 layers of Re and the $\mathrm{C}$ atoms adsorbed on the top layer. The bottom two layers of Re were kept fixed to their bulk geometry, and the other atoms in the simulation cell were fully relaxed. The surface geometry was defined using a supercell having the shape of a parallelogram, with unit vectors $7 \vec{a}_{\mathrm{Re}, 1}, 2 \vec{a}_{\mathrm{Re}, 1}+5 \vec{a}_{\mathrm{Re}, 2}$, and $10 \vec{a}_{\mathrm{Re}, 3}$ [with $\vec{a}_{\mathrm{Re}, 1,2,3}$ the $\operatorname{Re}(0001)$ unit vectors]. The length of $10 \vec{a}_{\mathrm{Re}, 3}$ ensured a vertical distance of about $20 \AA$ between the surface and the bottom layer of the periodic image of the slab, which is large enough to decouple the two, as demonstrated by tests using a longer $\vec{a}_{3}=15 \times \vec{a}_{\mathrm{Re}, 3}$, which showed essentially no energy difference. Brillouin-zone sampling was performed using a $2 \times 3 \times 1$ Monkhorst-Pack [44] grid. Tests with a $3 \times 5 \times 1$ grid showed differences of less than $0.2 \mathrm{meV}$ per $\mathrm{C}$ atom in the energy differences between different structures.

\section{RESULTS AND DISCUSSION}

\section{A. Optimization of graphene growth}

To selectively prepare graphene we used $P_{\mathrm{C}_{2} \mathrm{H}_{4}}=5 \times 10^{-7}$ mbar and 11 temperature cycles up to $970 \mathrm{~K}$ [Fig. 1(b)]. Such a temperature was found optimal for reaching the highest graphene structural quality, and is a limit above which graphene transforms into the surface carbide. Figure 1(a) shows a typical RHEED pattern of graphene-covered $\operatorname{Re}(0001)$. We observe streaks related to the average lattice structure of Re, graphene, and the moiré superlattice, the 

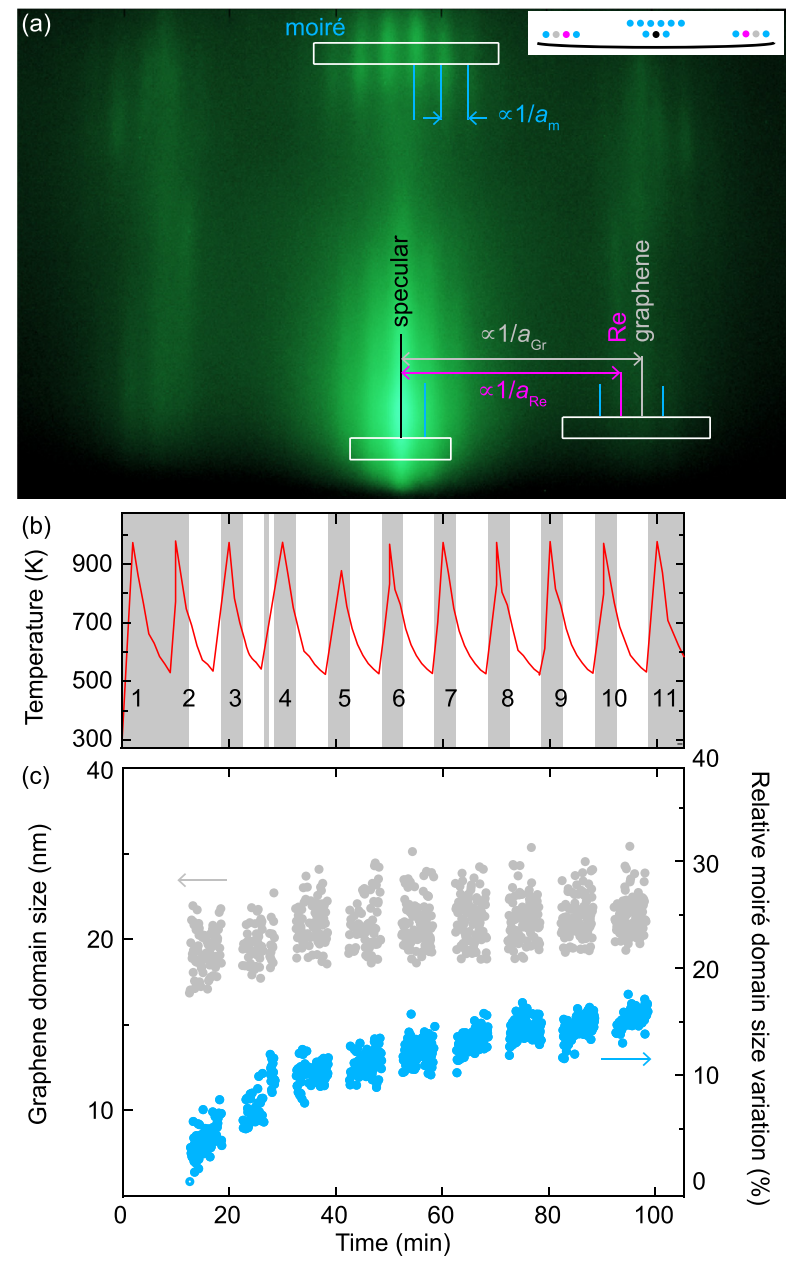

FIG. 1. (a) RHEED pattern of graphene-covered Re(0001) along the [0110] incident azimuth. Colored lines indicate the specular (black), Re (pink), graphene (gray), and moiré (blue) streaks (lines are superimposed only on the right side of the pattern). The distance between neighbor streaks is inversely proportional to the lattice parameter. Regions of interest are indicated with white rectangles. Inset shows a schematic top view of the reciprocal space with all observed rods and a cut of the Ewald sphere (black line). (b) Sample temperature as a function of time, during the growth process consisting of 11 temperature cycles. The first and eleventh cycles were not included in our structural analysis, and the RHEED patterns falling in gray rectangles were excluded from the analysis [37]. (c) Sizes of the structurally coherent domains in graphene and the moiré, as deduced by RHEED analysis as a function of time.

latter being a (quasi)coincidence lattice arising from the lattice mismatch between graphene and the $\operatorname{Re}(0001)$ substrate $[1,3]$.

To characterize the structure, a sequence of RHEED patterns was acquired as a function of growth time. Distinct regions of interest were selected in the RHEED patterns [Fig. 1(a)] to specifically address the graphene lattice and the moiré contributions.

In our analysis, the Re lattice parameter is assumed constant, unaltered by the presence of graphene (local strain possibly induced by graphene should not alter the average Re lattice parameter) or a few $100 \mathrm{~K}$ changes of temperature during the temperature cycles (which should induce $0.3 \%$ lattice thermal contraction/expansion [45], i.e., smaller than the $\simeq 1 \%$ uncertainty on our extracted strain values).

No change in the average graphene lattice parameter (graphene streak position) is observed (see Fig. S3 in the SM). Using the Re lattice parameter (streak position) as a reference, we find a $2.46 \AA$ lattice parameter, close to the literature values. Consistent with this analysis, the moiré superlattice parameter is essentially constant, of $2.14 \mathrm{~nm}$ (see Fig. S3 and related discussion in the $\mathrm{SM}$ ).

Structural changes are more obvious in the size of the structurally coherent (i.e., scattering coherently in the RHEED experiments) graphene and moiré domains. Within a simple kinematic theory of electron diffraction the size of structurally coherent domains (see discussion below) is inversely proportional to the width of the scattering rods perpendicular to the surface [46], i.e., to the FWHM of the RHEED streaks.

The FWHM of the Re streak (not shown) is unchanged from cycle 2 to cycle 10 and amounts to $\simeq 0.40 \AA^{-1}$. Assuming that Re is structurally coherent over a size of $100 \mathrm{~nm}$, the average size of the $\operatorname{Re}(0001)$ terraces, we expect a FWHM $\simeq 2 \pi /$ size $=0.006 \AA^{-1}$. The observed larger FWHM is dominated by instrumental effects, especially the coherence length of the electron beam (see discussion in the SM), which is about $20 \mathrm{~nm}$ in the first Laue zone, where the modulus of the out-of-plane scattering vector is the shortest [close to the bottom horizontal in Fig. 1(a)]. By analyzing the Re streak we extract the contribution of these instrumental effects, which also intervene in the FWHM of the graphene and moiré streaks.

Figure 1(c) shows the size of structurally coherent graphene domains, extracted from the FWHM of the corresponding streaks after the contribution of instrumental effects has been removed. This size first increases by about $20 \%$ from cycle $2(19 \mathrm{~nm})$ to $4(22 \mathrm{~nm})$. From cycle 4 , the coherent domain size saturates. As shown below, this saturation is inherent to the measurement itself, and due to the limited coherence of the electron beam in the first Laue zone, which is precisely of the order of $20 \mathrm{~nm}$.

We turn to the analysis of the FWHM of the moire streaks. Such streaks are found in the first Laue zone around the graphene and Re streaks, but there, their low intensity (hence signal-to-noise ratio) prevents us from identifying any trend such as the one observed for the graphene streaks, because of the strong dispersion of the data. This is why we also analyzed other moiré streaks, positioned above the specular streak in Fig. 1(a), in the second Laue zone. For these streaks the FWHM is globally smaller due to an effectively larger coherence length of the electron beam. This means that we cannot extract absolute values of the domain sizes here (this would require us to assess the contribution of instrumental effects using, e.g., a Re streak in this particular region of reciprocal space, which we cannot do). Nevertheless, we can use relative units to accurately describe the variation of their FWHM. Figure 1(c) reveals a clear increase of the moiré domain size from cycle 2 to 10 , of about $15 \%$. While the increase is stronger for the first 3-4 cycles, no saturation such as the one observed for the graphene streaks is observed here.

Our RHEED observations are consistent with LEED measurements reported in Ref. [2], where the moiré diffraction 
spots were found to increase in intensity at each temperature cycle.

Within the simple kinematic theory, the broadening of the graphene and moiré streaks/spots (and the reduction of their intensity) is due to the finite size of the structurally coherent domains. The expression "structurally coherent domains" refers to either actual graphene flakes or regions within an extended layer where the atomic lattice is essentially undistorted - in both cases, the domains that produce the constructive interferences at the origin of the diffraction streaks. To tell which case is relevant here, we use qualitative arguments provided by an STM analysis.

Figures 2(a) and 2(b) show that the graphene coverage increases only marginally between the first and second cycle. To understand why this is so, we note that after the first temperature cycle, the sample has been exposed to a large dose of typically $100 \mathrm{~L}$ of ethylene at temperatures above $700 \mathrm{~K}$. Since at these elevated temperatures ethylene molecules should be efficiently cracked by the $\operatorname{Re}(0001)$ surface, the observed close-to- $100 \%$ graphene coverage is not a surprise. This suggests that the effect limiting the structural coherency of graphene should be lattice distortions (strains).

Although the STM images shown in Fig. 2 are not atomically resolved (hence they do not directly reveal strains), they reveal the moiré pattern. Strains in the carbon lattice reflect in the moiré lattice, where they are amplified [47], here by a factor of about 10. Strong distortions are indeed observed in the STM images for the first two cycles [Figs. 2(a) and 2(b); even more so after the first cycle, see Fig. 2(a)]: the moiré hills (regions of larger apparent height) are positioned onto a distorted lattice and they occasionally or often appear elongated or compressed. In addition to these deformations, defects occurring at local scale (and as such not liable to broaden the diffraction streaks) are also observed. In particular, so-called vacant moiré hills, corresponding to stacking faults in either the metal or graphene lattice [48,49], are found. As the number of temperature cycles increases, the number of distortions and local defects is reduced [Figs. 2(a)-2(c)]. Consistent with these real-space observations and with the RHEED data, the Fourier transforms of the STM images (insets in Fig. 2) exhibit better-defined and more numerous harmonics as the number of cycles increases. The progressive ordering of the graphene and moiré (super)lattices as a function of the number of cycles may occur via the rotation of less-energetically-favorable domains, the incorporation of carbon nanoclusters [50], and the release of heterogeneous strain in graphene (for instance through local sliding of the graphene lattice with respect to that of the substrate or through the healing of point defects such as vacancies [51]).

Obviously, increasing the number of temperature cycles has positive effects on the quality of graphene grown on $\operatorname{Re}(0001)$. Increasing the time of the final annealing, even at lower temperature, does not have the same effects, and instead transforms graphene into a surface rhenium carbide.

\section{B. STM and RHEED observations of the rhenium surface carbide}

To promote the formation of the surface carbide, we found that decreasing $P_{\mathrm{C}_{2} \mathrm{H}_{4}}$ to $1 \times 10^{-7}$ mbar during the temperature
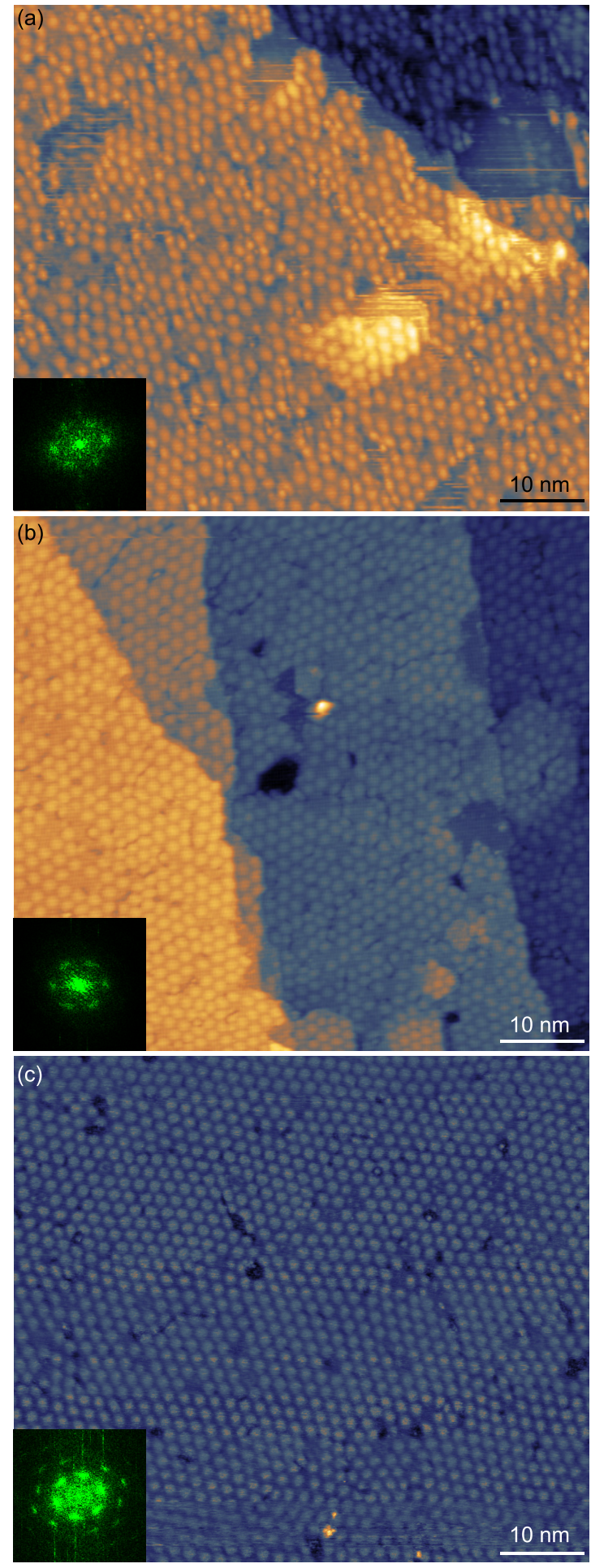

FIG. 2. STM images ( $4 \mathrm{nA}, 2 \mathrm{nA}, 1 \mathrm{nA} ; 0.01 \mathrm{~V}, 1 \mathrm{~V}, 0.6 \mathrm{~V})$ of graphene-covered $\operatorname{Re}(0001)$ after one (a), two (b), and 10 (c) temperature cycles. The corresponding Fourier transforms are shown as insets.

cycles and decreasing the temperature of the annealing steps to $870 \mathrm{~K}$ are well suited. After four temperature cycles and a fifth one in the absence of $\mathrm{C}_{2} \mathrm{H}_{4}$, the sample was finally annealed for $1 \mathrm{~h}$ at $870 \mathrm{~K}$.

In the RHEED patterns, additional streaks are observed compared to the previous sample (where only graphene was 

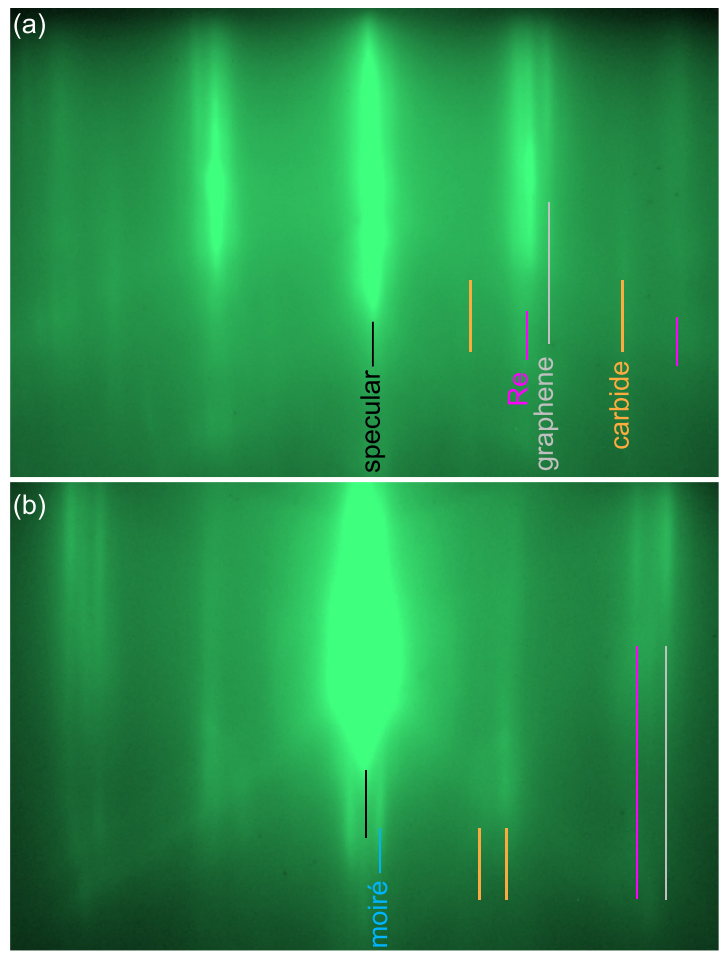

FIG. 3. RHEED patterns of coexisting graphene and surface carbide phases along the (a) [11 20$]$ and (b) [0110] incident azimuths. Vertical colored lines indicate the specular (black), Re (pink), graphene (gray), moiré (blue), and surface carbide (orange) streaks.

formed). Along two different azimuths, two new characteristic streaks are observed (Fig. 3). These streaks correspond to the intersection of the Ewald sphere with scattering rods extending perpendicular to the surface. The footprint of these scattering rods in the surface plane was observed in previous LEED data [2,36] (see Fig. S4 in the SM). These LEED patterns were attributed to a surface rhenium carbide with a $(7 \times \sqrt{19})$ unit cell, i.e., with lower symmetry than the $\operatorname{Re}(0001)$ surface, and appearing in the form of domains with different crystallographic orientations.

Figure 4(a) shows a representative STM image of the surface. Besides the graphene patches (easily recognized from their moiré patterns), isolated clusters assigned to carbon clusters of well-defined size $[28,48]$ and bunches of parallel lines separated by $0.5 \mathrm{~nm}$ are observed. The latter structure covers about $30 \%$ of the surface in the preparation conditions we have chosen and accounts for our RHEED observations (see below). We hence ascribe it to the surface rhenium carbide.

\section{Orientation variants of the surface carbide and symmetry considerations}

The STM images make it obvious that the surface carbide has several well-defined crystallographic orientations [Fig. 4(a)]. This is consistent with previous LEED observations [2,36] (also see Figs. S4 and S5 in the SM), which were interpreted by invoking the coexistence of six kinds of domains [2].

The possible $(7 \times \sqrt{19})$ unit cells of each of these six domains are sketched in Fig. 5(a) (right panel) for one specific terrace. They consist of three pairs, related to one another by $120^{\circ}$ rotations about the $C_{3}$ symmetry axis standing perpendicular to the surface. Since there are two possible kinds of terraces on $\operatorname{Re}(0001)$ according to its hexagonal compact stacking, a total of 12 different kinds of carbide domains is expected in STM. In the left panel of Fig. 5(a), one kind of pair is sketched on either side of a $\operatorname{Re}(0001)$ atomic step
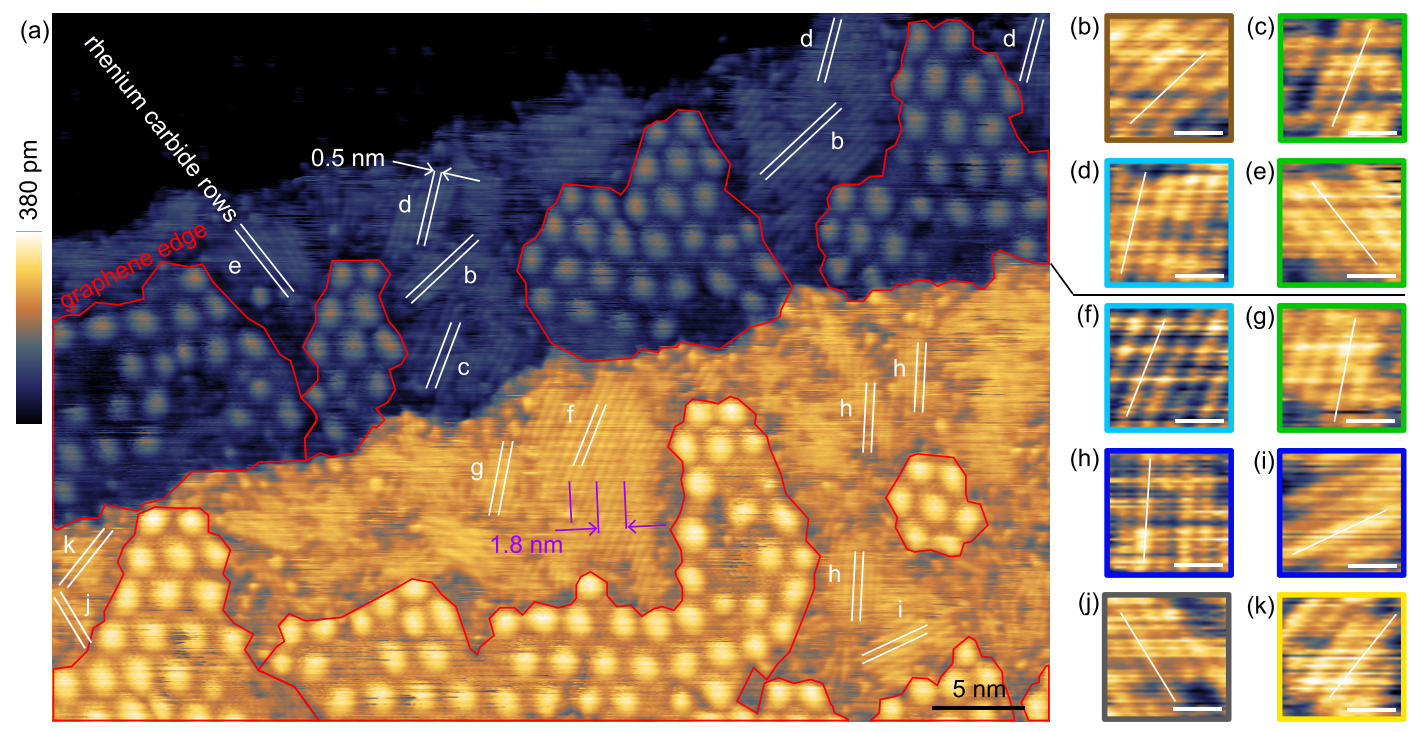

FIG. 4. (a) STM images (2 nA, 1 V) of a Re(0001) surface with coexisting graphene and surface carbide. Carbide variants with different orientations are marked with letters $b-k$. Purple lines highlight the modulation of apparent height observed on certain variants of the carbide phase. (b)-(k) Close-up views (scale bars, $1 \mathrm{~nm}$ ) of the ten (out of 12 possible; $b, d, h$ are observed several times) carbide phase variants, each with a distinct crystallographic orientation, identified in (a), where (b)-(e) are the variants identified in the lower terrace, and (f)-(k) are the variants identified in the upper terrace. Equivalent variants (due to the surface symmetry or on distinct terraces) are signaled with a single color of the frame around the panels. 
(a)

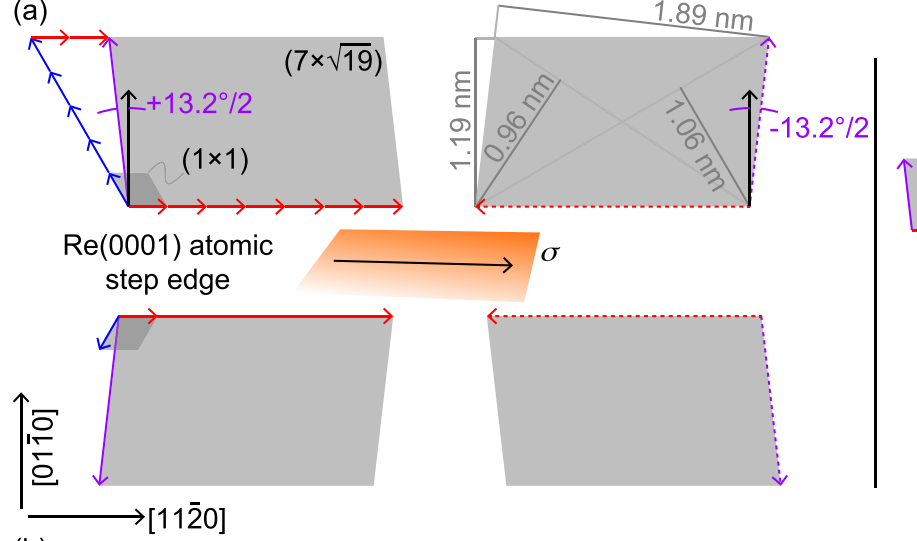

(b)

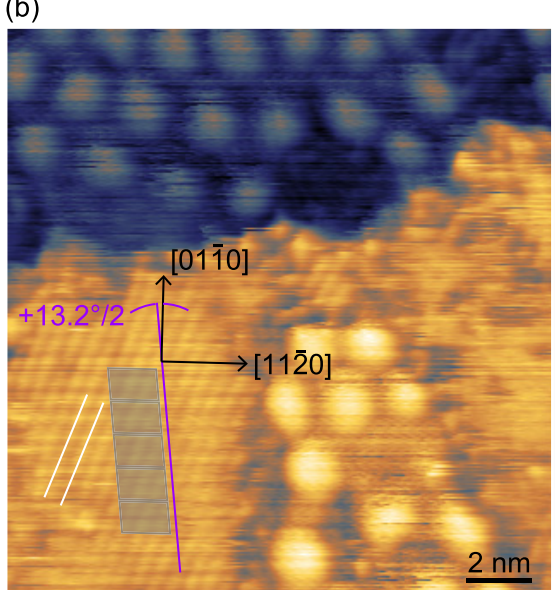

(d)
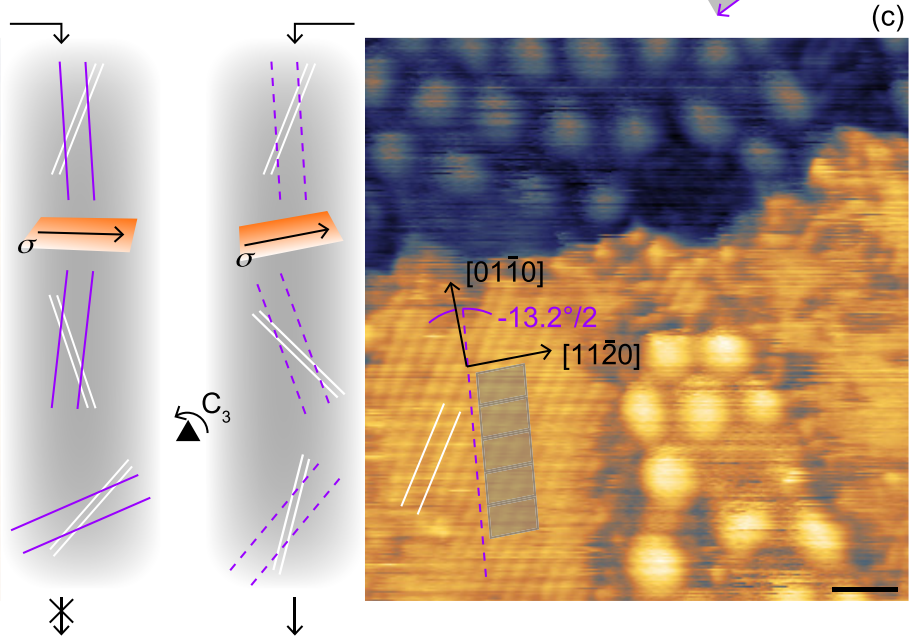

(c)

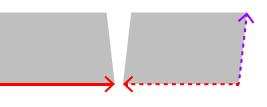

$\mathrm{A}_{\mathrm{C}_{3}}$

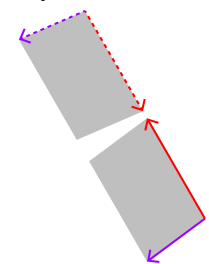

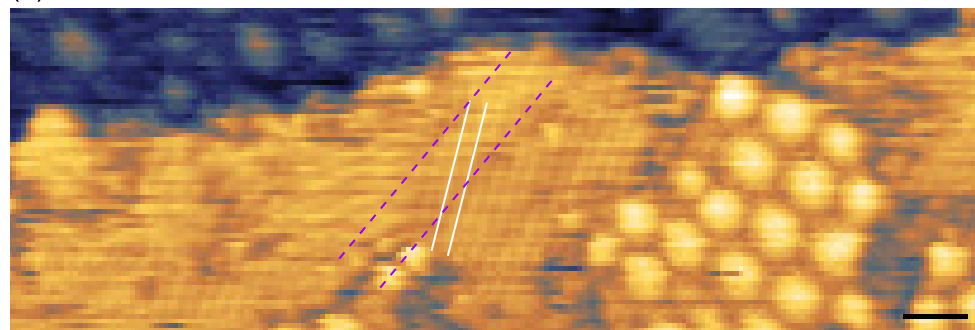

FIG. 5. Symmetry of the carbide's lattice in its different variants. (a) On a given Re(0001) terrace, there are two kinds (solid and dotted arrows) of $(7 \times \sqrt{19})$ unit cells [large gray parallelograms constructed from the $\operatorname{Re}(0001)$ unit cell shown with a small gray $(1 \times 1)$ rhombus], each reflected with respect to the corresponding unit cell in the neighbor Re(0001) terrace (left). Each of the two kinds of unit cells on each terrace has two other equivalent unit cells, according to the $C_{3}$ symmetry of the surface (right). (b), (c) Close-up views of Fig. 4(a). The $(7 \times \sqrt{19})$ unit cells (gray parallelograms) fit in between two purple lines that mark the apparent modulation along the line pattern of the carbide. Two possible variants are considered in (b) and (c), each corresponding to a different orientation of the [11 $\overline{2} 0]$ Re direction. (d) STM image $(2 \mathrm{nA}, 1 \mathrm{~V})$ of a carbide variant on a terrace adjacent to the one of the carbides in (b) and (c). At the center, the carbide pattern is mirrored with respect to a $\sigma$ plane (step edge) containing the [11 20$]$ direction and then rotated about a $C_{3}$ symmetry axis (surface symmetry). The resulting patterns match for (c) and (d).

edge. The symmetry operation relating the unit cells on the left and right terraces is a mirror $(\sigma)$ plane containing a $\langle 11 \overline{2} 0\rangle$ direction of $\operatorname{Re}(0001)$. To complete the description, we turn to the two unit cells of each pair (on each terrace): they are related to one another by a mirror operation, about a plane containing a $\langle 01 \overline{1} 0\rangle$ direction of $\operatorname{Re}(0001)$. While one of them has unit cell vectors of $7 \vec{a}_{\mathrm{Re}, 1}$ and $2 \vec{a}_{\mathrm{Re}, 1}+$ $5 \vec{a}_{\mathrm{Re}, 2}$ [solid arrows in Fig. 5(a), left], for the other the unit cell vectors are $-7 \vec{a}_{\mathrm{Re}, 1}$ and $3 \vec{a}_{\mathrm{Re}, 1}+5 \vec{a}_{\mathrm{Re}, 2}$ [dotted arrows in Fig. 5(a), left]. The vectors $2 \vec{a}_{\mathrm{Re}, 1}+5 \vec{a}_{\mathrm{Re}, 2}$ and $3 \vec{a}_{\mathrm{Re}, 1}+$
$5 \vec{a}_{\mathrm{Re}, 2}$ form a $\pm 13.2^{\circ} / 2$ angle with the [01ㅣㅣ direction [52].

We wish to determine the unit cells [among those presented in Fig. 5(a)] of the variants identified in Fig. 4. To do so, we must first unambiguously identify the above-mentioned mirror planes, i.e., the substrate crystallographic directions

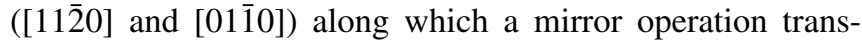
forms the unit cell of a domain into a unit cell of another kind of domain on the same $\operatorname{Re}(0001)$ terrace or on an adjacent terrace. The bare $\operatorname{Re}(0001)$ surface is however not directly 
accessible here; hence it is not possible to directly identify these directions with atomic resolution imaging. The substrate's crystallographic directions cannot be identified indirectly either from the moiré pattern crystallographic directions in the neighbor graphene patches, since their orientation is known to be significantly scattered (it actually amplifies the rotations of the graphene lattice with respect to the metal lattice by a factor 10 [47]).

More useful is the orientation of a feature that we have overlooked so far in the STM images. A modulation is observed (highlighted with purple lines) in the apparent height of the linear pattern of the surface carbide [Fig. 5(b), corresponding to a zoom on the variant labeled $f$ in Fig. 4(a)]. The periodicity of this modulation is $1.8 \mathrm{~nm}$, which is very close to the $1.89 \mathrm{~nm}$ value for the long period of a tiling with the $(7 \times \sqrt{19})$ unit cell [Fig. 5(a)]. As shown in Figs. 5(a)-5(c), this match suggests that the [01 10$]$ direction of $\operatorname{Re}(0001)$ is either $+13.2^{\circ} / 2$ or $-13.2^{\circ} / 2$ off the direction of the purple line; i.e., the purple line aligns the unit cell vector $2 \vec{a}_{\mathrm{Re}, 1}+5 \vec{a}_{\mathrm{Re}, 2}$ $[(7 \times \sqrt{19})$ unit cell with solid vectors in Fig. 5(a)] or the unit cell vector $3 \vec{a}_{\mathrm{Re}, 1}+5 \vec{a}_{\mathrm{Re}, 2}[(7 \times \sqrt{19})$ unit cell with dotted vectors in Fig. 5(a)].

To determine which of the two $\operatorname{Re}(0001)$ crystallographic orientations $\left( \pm 13.2^{\circ} / 2\right)$ is the actual one, we compare the two rhenium carbide domains of kind $f$ located on the two adjacent terraces (separated by an atomic substrate step edge) imaged in Figs. 5(b) and 5(c). For each of the two $\operatorname{Re}(0001)$ crystallographic configurations $\left( \pm 13.2^{\circ} / 2\right)$, we applied a mirror operation to the carbide's pattern, with a $\sigma$ mirror plane containing the $[11 \overline{2} 0] \operatorname{Re}(0001)$ direction. This operation "transfers" the orientation of the carbide's pattern to the other terrace. After this operation, only one configuration [Fig. 5(c)] yields the same orientation (modulo $120^{\circ}$ ) for the two domains, which hence identifies the actual [11 20$]$ $\operatorname{Re}(0001)$ direction. Note that this orientation matches the average orientation of the substrate step edge [53]. Given the high temperature used to prepare the $\operatorname{Re}(0001)$ surface, this might not be accidental: such a step edge orientation is energetically more favorable.

Now, the carbide domains can be categorized in families, each with a specific kind of unit cell shown in Fig. 4(a). Variants are grouped via $120^{\circ}$ rotations within a terrace, and via mirror symmetries along the [1120] directions of the $\operatorname{Re}(0001)$ between terraces. Overall, we find six families of equivalent atomic arrangements. They are highlighted with different colors in Figs. 4(b)-4(k).

\section{Possible atomic structures for the motif of the rhenium carbide}

Beyond these symmetry considerations, the atomic details of the surface carbide remain essentially unknown. Although the STM images presented in Figs. 4 and 5 reveal an atomic-scale contrast, they do not directly unveil the atomic arrangement of rhenium carbide, but essentially reveal lines (0.5 nm separation) with a specific crystallographic orientation and a height modulation pattern (1.8 nm period) [54]. Former rhenium core level photoemission data together with DFT calculations [2] indicate that the lines actually consist of rows of specific kinds of trimers of $\mathrm{C}$ atoms. These trimers consist of $\mathrm{C}$ atoms bonded to so-called fcc and hep hollow sites of $\operatorname{Re}(0001)$, sitting on top of a $\operatorname{Re}$ atom and a hollow site in the second Re layer, respectively. The core level data also revealed a close-to- $50 \%$ weight of the $\mathrm{C}$ atoms on these two binding sites [2].

We were able to produce four tentative structures compatible with all these experimental facts; three have a unique trimer crystallographic orientation, and one features trimers with two kinds of crystallographic orientations. All these structures have one half of the $\mathrm{C}$ atoms occupying hcp sites (and the other half the fcc sites). Their composition can be described as $\operatorname{Re}_{11} \mathrm{C}_{8}$, when considering the number of hollow sites of the $\operatorname{Re}(0001)$ surface that are occupied by $\mathrm{C}$ atoms. We optimized the atomic positions in these structures with the help of DFT calculations (Fig. 6(a) and Fig. S7 in the SM [37]). Obvious distortions are seen within each unit cell: on top and side views (Figs. 6(a)-6(c) and Fig. S7 in the SM [37]), the $\mathrm{C}$ trimers do not all look the same, and the Re atoms are displaced (by several $1 \%$ both within the surface plane and perpendicular to it), most prominently in the topmost $\mathrm{Re}$ layer.

The DFT calculations show that all the structures are stable. We first focus on the optimized structure shown in Fig. 6(a), where the hcp-fcc-hcp trimers align along segments that span across three $(7 \times \sqrt{19})$ unit cells. These segments have exactly the same crystallographic orientation as those highlighted with white lines for the variant labeled $f$ in Fig. 4(a). At the end of these segments, the atomic C density is locally lower, providing a possible interpretation for the observed 1.8-nm-periodic modulation of the apparent height highlighted with purple lines for this variant in Figs. 4(a) and 5(b)-5(d). Why such (rather complex) atomic configurations are selected during growth is an open question, whose answer is beyond the scope of the present work: they should correspond to an energy minimum of the system, either a local or a global one (depending on kinetic hindrances), which to some extent mitigates elastic deformations in, e.g., the substrate and the $\mathrm{C}$ trimers, repulsive $\mathrm{C}-\mathrm{C}$ interactions mediated by the substrate [50], etc. Overall, the constant-height cut in the DFT-simulated electronic density map corresponding to this structure [Fig. 6(d)] appears compatible [55] with our STM observations of this variant.

The energy of the structure we just discussed is higher by $0.9 \mathrm{eV}$ per unit cell than the one of the lowest-energy structures (Fig. S6 in the SM [37]). Altogether, the four structures that were optimized with DFT differ by 0.4 to $1.1 \mathrm{eV}$ per unit cell. Expressed relative to the number of $\mathrm{C}$ atoms in each unit cell, these energy differences only amount to a few millielectron-volts, which are small values compared to thermal energy (a few to several $10 \mathrm{meV}$ ) in the growth and STM imaging conditions. It is hence reasonable to expect that the different models we propose in Fig. S6 in the SM [37] actually each correspond to different kinds of variants observed for instance in Fig. 4. Interestingly, the binding energy per $\mathrm{C}$ atom in the carbide structures we have considered are about $500 \mathrm{meV}$ lower than that of $\mathrm{C}$ atoms in graphene/ $\operatorname{Re}(0001)$, which hence is predicted to be the thermodynamically stable form of $\mathrm{C}$. Beyond the scope of the present work and as already mentioned in the introduction, it would certainly be interesting to understand the influence of the $\mathrm{C}$ 

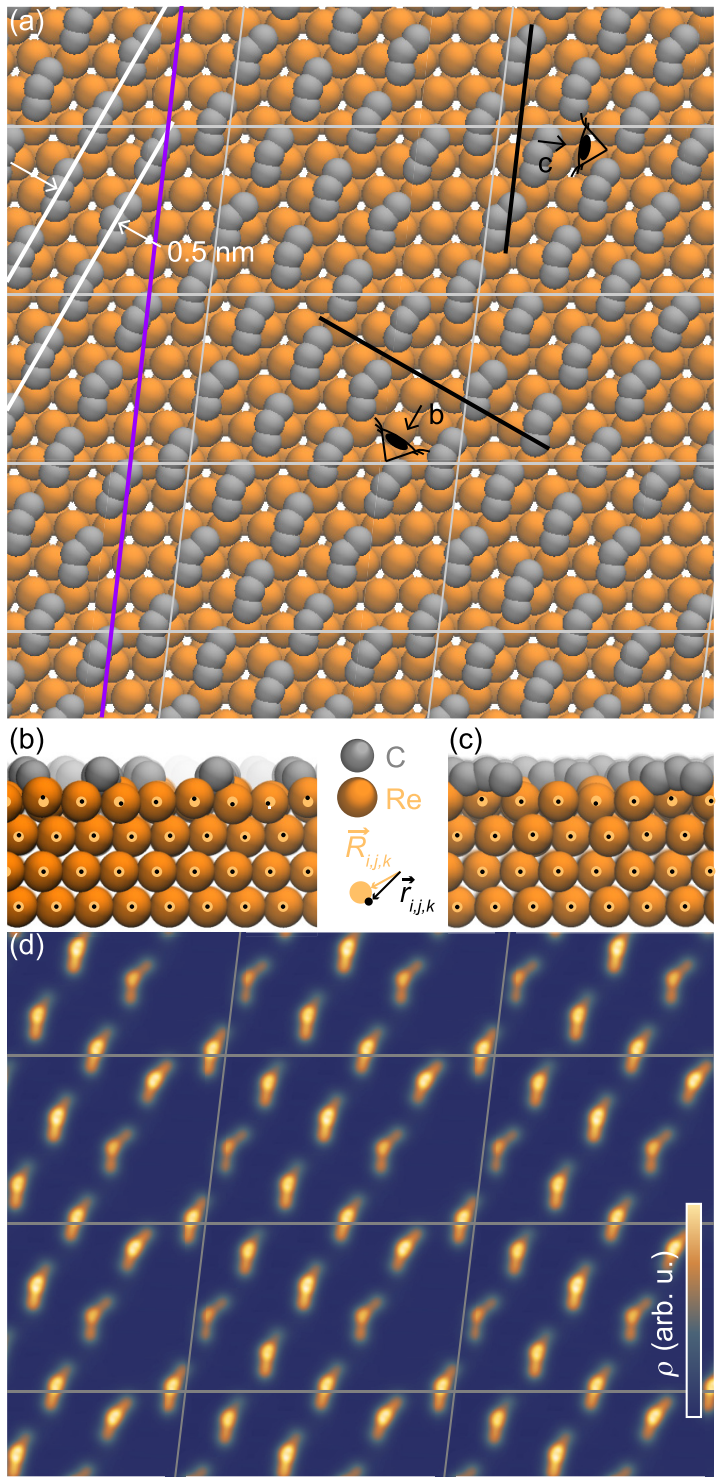

FIG. 6. (a) Atomic details of the lattice motif of the second lowest energy structure of a surface rhenium carbide variant labeled $f$ in Fig. 4(a), optimized by DFT calculations. (b), (c) Cross sections along the two directions marked in (a). The orange and black dots mark the positions $\vec{R}_{i, j, k}$ and $\vec{r}_{i, j, k}$ of Re atoms (labeled by indexes $i, j, k)$ in an undistorted lattice and in the actual lattice, respectively. (d) Cut (0.6 A higher than the average height of topmost atoms) in the corresponding calculated electronic density $(\rho)$ map.

chemical potential at the surface of $\operatorname{Re}(0001)$, and in particular of the excess chemical potential with respect to the critical value corresponding to graphene and carbide nucleation. The relative stability of the different $\mathrm{C}$ phases may depend, quantitatively if not qualitatively, on this excess value. Please also note that while our calculations are performed for a surface carbide structure extending to infinity (fully periodic system), our experiments reveal variants in the form of domains with finite extension in the range of 5 to $10 \mathrm{~nm}$ [Fig. 4(a)]. There, finite-size and boundary effects may alter the energy hierarchy between the different structures and phases we considered.

\section{CONCLUSION}

We have investigated the structure of graphene and a surface carbide grown on the (0001) surface of Re, using RHEED, STM, and DFT calculations. We find that the number of cycles used to grow graphene is a key parameter to improve its structural quality. On the contrary, long annealing times, which could give time for the system to heal its defects, do not have the desired effect. They promote the transformation of graphene into a surface carbide with a $(7 \times \sqrt{19})$ unit cell. We were able to identify the possible crystallographic orientations (variants) of two sixfold families of equivalent domains of the rhenium surface carbide. The carbide is highly anisotropic, consisting of parallel lines separated by $0.5 \mathrm{~nm}$, and periodically modulated at the nanometer scale. We have proposed a realistic atomistic model of one of the carbide variants, whereby the observed lines consist of eight adjacent carbon trimers, in agreement with previous photoemission data. In a $\mathrm{C}$-Re phase diagram, the rhenium carbide would represent a rather low-density $\mathrm{C}$ phase, in this sense intermediate between graphene and a dilute phase [28] in the Re bulk. We suggest that the surface rhenium carbide could serve as a template for the self-organization of functional nanoclusters or molecules.

\section{ACKNOWLEDGMENTS}

This work was supported by the Région Rhône Alpes (ARC6 program) and the Labex LANEF. Funding from the French National Research Agency under the 2DTransformers (OH-RISQUE program Grant No. ANR-14-OHRI-0004) and ORGANI'SO (Grant No. ANR-15-CE09-0017) projects is gratefully acknowledged. M.P. and D.A. are supported by the Natural Environment Research Council Grant No. NE/R000425/1. DFT calculations were performed on the Monsoon2 system, a collaborative facility supplied under the Joint Weather and Climate Research Programme, a strategic partnership between the UK Met Office and the Natural Environment Research Council.
[1] E. Miniussi, M. Pozzo, A. Baraldi, E. Vesselli, R. R. Zhan, G. Comelli, T. O. Menteş, M. A. Niño, A. Locatelli, S. Lizzit, and D. Alfè, Thermal Stability of Corrugated Epitaxial Graphene Grown on Re(0001), Phys. Rev. Lett. 106, 216101 (2011).

[2] E. Miniussi, M. Pozzo, T. Menteş, M. Niño, A. Locatelli, E. Vesselli, G. Comelli, S. Lizzit, D. Alfè, and A. Baraldi, The competition for graphene formation on $\operatorname{Re}(0001)$ : A complex interplay between carbon segregation, dissolution and carburisation, Carbon 73, 389 (2014).

[3] C. Tonnoir, A. Kimouche, J. Coraux, L. Magaud, B. Delsol, B. Gilles, and C. Chapelier, Induced Superconductivity in Graphene Grown on Rhenium, Phys. Rev. Lett. 111, 246805 (2013). 
[4] M. Papagno, P. Moras, P. M. Sheverdyaeva, J. Doppler, A. Garhofer, F. Mittendorfer, J. Redinger, and C. Carbone, Hybridization of graphene and a Ag monolayer supported on Re(0001), Phys. Rev. B 88, 235430 (2013).

[5] L. Gao, Y. Liu, R. Shi, T. Ma, Y. Hu, and J. Luo, Influence of interface interaction on the moiré superstructures of graphene on transition-metal substrates, RSC Adv. 7, 12179 (2017).

[6] B. Wang, M.-L. Bocquet, S. Marchini, S. Günther, and J. Wintterlin, Chemical origin of a graphene moiré overlayer on Ru(0001), Phys. Chem. Chem. Phys. 10, 3530 (2008).

[7] S. Marchini, S. Günther, and J. Wintterlin, Scanning tunneling microscopy of graphene on $\mathrm{Ru}(0001)$, Phys. Rev. B 76, 075429 (2007).

[8] P. W. Sutter, J.-I. Flege, and E. A. Sutter, Epitaxial graphene on ruthenium, Nat. Mater. 7, 406 (2008).

[9] E. Sutter, D. Acharya, J. Sadowski, and P. Sutter, Scanning tunneling microscopy on epitaxial bilayer graphene on ruthenium (0001), Appl. Phys. Lett. 94, 133101 (2009).

[10] B. Wang, M. Caffio, C. Bromley, H. Früchtl, and R. Schaub, Coupling epitaxy, chemical bonding, and work function at the local scale in transition metal-supported graphene, ACS Nano 4, 5773 (2010).

[11] Y. Gamo, A. Nagashima, M. Wakabayashi, M. Terai, and C. Oshima, Atomic structure of monolayer graphite formed on Ni(111), Surf. Sci. 374, 61 (1997).

[12] I. Pletikosić, M. Kralj, P. Pervan, R. Brako, J. Coraux, A. T. N'Diaye, C. Busse, and T. Michely, Dirac Cones and Minigaps for Graphene on Ir(111), Phys. Rev. Lett. 102, 056808 (2009).

[13] C. Busse, P. Lazić, R. Djemour, J. Coraux, T. Gerber, N. Atodiresei, V. Caciuc, R. Brako, A. T. N'Diaye, S. Blügel, J. Zegenhagen, and T. Michely, Graphene on $\operatorname{Ir}(111)$ : Physisorption with Chemical Modulation, Phys. Rev. Lett. 107, 036101 (2011).

[14] H. Zi-Pu, D. Ogletree, M. Van Hove, and G. Somorjai, LEED theory for incommensurate overlayers: Application to graphite on Pt(111), Surf. Sci. 180, 433 (1987).

[15] T. Land, T. Michely, R. Behm, J. Hemminger, and G. Comsa, STM investigation of single layer graphite structures produced on Pt(111) by hydrocarbon decomposition, Surf. Sci. 264, 261 (1992).

[16] P. Sutter, J. T. Sadowski, and E. Sutter, Graphene on Pt(111): Growth and substrate interaction, Phys. Rev. B 80, 245411 (2009).

[17] L. Gao, J. R. Guest, and N. P. Guisinger, Epitaxial graphene on $\mathrm{Cu}(111)$, Nano Lett. 10, 3512 (2010).

[18] B. Kiraly, E. V. Iski, A. J. Mannix, B. L. Fisher, M. C. Hersam, and N. P. Guisinger, Solid-source growth and atomic-scale characterization of graphene on $\operatorname{Ag}(111)$, Nat. Commun. 4, 1 (2013).

[19] S. Nie, N. C. Bartelt, J. M. Wofford, O. D. Dubon, K. F. McCarty, and K. Thürmer, Scanning tunneling microscopy study of graphene on $\mathrm{Au}(111)$ : Growth mechanisms and substrate interactions, Phys. Rev. B 85, 205406 (2012).

[20] P. Merino, M. Švec, A. L. Pinardi, G. Otero, and J. A. Martín-Gago, Strain-driven moiré superstructures of epitaxial graphene on transition metal surfaces, ACS Nano 5, 5627 (2011).

[21] E. Loginova, S. Nie, K. Thürmer, N. C. Bartelt, and K. F. McCarty, Defects of graphene on $\operatorname{Ir}(111)$ : Rotational domains and ridges, Phys. Rev. B 80, 085430 (2009).
[22] H. Hattab, A. N'Diaye, D. Wall, G. Jnawali, J. Coraux, C. Busse, R. van Gastel, B. Poelsema, T. Michely, F.-J. Meyer zu Heringdorf, and M. Horn-von Hoggen, Growth temperature dependent graphene alignment on $\operatorname{Ir}(111)$, Appl. Phys. Lett. 98, 141903 (2011).

[23] A. Artaud, L. Magaud, T. Le Quang, V. Guisset, P. David, C. Chapelier, and J. Coraux, Universal classification of twisted, strained and sheared graphene moiré superlattices, Sci. Rep. 6, 25670 (2016).

[24] H. Ago, Y. Ito, N. Mizuta, K. Yoshida, B. Hu, C. M. Orofeo, M. Tsuji, K. Ikeda, and S. Mizuno, Epitaxial chemical vapor deposition growth of single-layer graphene over cobalt film crystallized on sapphire, ACS Nano 4, 7407 (2010).

[25] P. Sutter, P. Albrecht, and E. A. Sutter, Graphene growth on epitaxial Ru thin films on sapphire, Appl. Phys. Lett. 97, 213101 (2010).

[26] T. Iwasaki, H. J. Park, M. Konuma, D. S. Lee, J. H. Smet, and U. Starke, Long-range ordered single-crystal graphene on highquality heteroepitaxial $\mathrm{Ni}$ thin films grown on $\mathrm{MgO}(111)$, Nano Lett. 11, 79 (2011).

[27] E. Koren, E. Sutter, S. Bliznakov, F. Ivars-Barcelo, and P. Sutter, Isolation of high quality graphene from $\mathrm{Ru}$ by solution phase intercalation, Appl. Phys. Lett. 103, 121602 (2013).

[28] N. Gall, S. Mikhailov, E. Rut'kov, and A. Y. Tontegode, Carbon interaction with the rhenium surface, Surf. Sci. 191, 185 (1987).

[29] G. Dong, D. W. van Baarle, M. Rost, and J. Frenken, Graphene formation on metal surfaces investigated by in-situ scanning tunneling microscopy, New J. Phys. 14, 053033 (2012).

[30] Y. Qi, C. Meng, X. Xu, B. Deng, N. Han, M. Liu, M. Hong, Y. Ning, K. Liu, J. Zhao, Q. Fu, Y. Li, Y. Zhang, and Z. Liu, Unique transformation from graphene to carbide on $\operatorname{Re}(0001)$ induced by strong carbon-metal interaction, J. Am. Chem. Soc. 139, 17574 (2017).

[31] J. Lahiri, T. Miller, L. Adamska, I. I. Oleynik, and M. Batzill, Graphene growth on $\mathrm{Ni}(111)$ by transformation of a surface carbide, Nano Lett. 11, 518 (2011).

[32] E. Mazaleyrat, S. Vlaic, A. Artaud, L. Magaud, T. Vincent, A. C. Gómez-Herrero, S. Lisi, P. Singh, N. Bendiab, V. Guisset, P. David, S. Pons, D. Roditchev, C. Chapelier, and J. Coraux, How to induce superconductivity in epitaxial graphene via remote proximity effect through an intercalated gold layer, 2D Mater. 8, 015002 (2020).

[33] J. Bansmann, L. Lu, V. Senz, A. Bettac, M. Getzlaff, and K. H. Meiwes-Broer, Structure and magnetism of self-organized Co islands, Eur. Phys. J. D 9, 461 (1999).

[34] A. Varykhalov, O. Rader, and W. Gudat, Self-organization of one-dimensional Au nanowires on a surface carbide and lateral electron quantization, Phys. Rev. B 72, 241404(R) (2005).

[35] A. Varykhalov, O. Rader, and W. Gudat, Origin of Au nanostructures on tungsten surface carbides, Phys. Rev. B 77, 035412 (2008).

[36] R. S. Zimmer and W. D. Robertson, A carbon structure on the $\operatorname{Re}(0001)$ surface, Surf. Sci. 29, 230 (1972).

[37] See Supplemental Material at http://link.aps.org/supplemental/ 10.1103/PhysRevMaterials.4.124002 for details on the analysis of the RHEED image sequence, the instrumental width of the RHEED streaks, RHEED patterns of coexisting graphene and surface carbide phases, and alternative structures of one variant of the surface carbide. 
[38] P. Hohenberg and W. Kohn, Inhomogeneous electron gas, Phys. Rev. 136, B864 (1964).

[39] W. Kohn and L. J. Sham, Self-consistent equations including exchange and correlation effects, Phys. Rev. 140, A1133 (1965).

[40] J. P. Perdew, K. Burke, and M. Ernzerhof, Generalized Gradient Approximation Made Simple, Phys. Rev. Lett. 77, 3865 (1996).

[41] P. E. Blöchl, Projector augmented-wave method, Phys. Rev. B 50, 17953 (1994).

[42] G. Kresse and J. Furthmüller, Efficient iterative schemes for ab initio total-energy calculations using a plane-wave basis set, Phys. Rev. B 54, 11169 (1996).

[43] G. Kresse and D. Joubert, From ultrasoft pseudopotentials to the projector augmented-wave method, Phys. Rev. B 59, 1758 (1999).

[44] H. J. Monkhorst and J. D. Pack, Special points for Brillouinzone integrations, Phys. Rev. B 13, 5188 (1976).

[45] D. R. Lide, CRC Handbook of Chemistry and Physics, 88th ed. (Taylor and Francis Group, Boca Raton, 2008), pp. 12-201.

[46] A. Ichimiya and P. I. Cohen, Reflection High-Energy Electron Diffraction (Cambridge University Press, Cambridge, 2004).

[47] J. Coraux, A. T. N'Diaye, C. Busse, and T. Michely, Structural coherency of graphene on $\operatorname{Ir}(111)$, Nano Lett. 8, 565 (2008).

[48] A. Artaud, E. Mazaleyrat, G. D. Förster, C. Tonnoir, B. Gilles, P. David, V. Guisset, L. Magaud, F. Calvo, C. Chapelier, and J. Coraux, Depressions by stacking faults in nanorippled graphene on metals, 2D Mater. 7, 025016 (2020).

[49] P. Pochet, B. C. McGuigan, J. Coraux, and H. T. Johnson, Toward moiré engineering in $2 \mathrm{~d}$ materials via dislocation theory, Appl. Mater. Today 9, 240 (2017).
[50] A. Artaud, L. Magaud, K. Ratter, B. Gilles, V. Guisset, P. David, J. I. Martinez, J. A. Martín-Gago, C. Chapelier, and J. Coraux, Size-selective carbon clusters as obstacles to graphene growth on a metal, Nano Lett. 18, 4812 (2018).

[51] N. Blanc, F. Jean, A. V. Krasheninnikov, G. Renaud, and J. Coraux, Strains Induced by Point Defects in Graphene on a Metal, Phys. Rev. Lett. 111, 085501 (2013).

[52] The $13.2^{\circ}$ angle presumably corresponds to the $12^{\circ}$ value assessed by LEED in a previous report [2].

[53] Also note that this assignment is consistent with the crystallographic orientation that can be deduced from a RHEED search for the main crystallographic directions as a function of the azimuthal angle. In our experimental setup unfortunately, absolute measurements of this angle are not precise, due to a significant nonreproducible backlash in the azimuthal rotation axis. We can only assess the absolute azimuthal angle via a visual inspection of the orientation of the sample with respect to the electron beam axis, which is no better than $\pm 10^{\circ}$.

[54] Due to the very demanding STM imaging conditions (the uneven surface, with a coexistence of graphene, carbide, and other adsorbed species, leads to frequent modifications of the STM probe apex), we are unable to collect higher resolution data than in Figs. 4 and 5, or a broader set of data allowing us to perform, for all domains observed in STM, the analysis we performed for domains of type $f$ (Fig. 4).

[55] Please keep in mind that our calculations, which do not include the effect of the STM tip, are better resolved than the experimental STM images. 